\title{
The Shortest Path of Touring Lines given in the Plane
}

\author{
Lijuan Wang ${ }^{1,2}$, Dandan $\mathrm{He}^{2, *}$, Ansheng Deng ${ }^{1}$ and Tao Ning ${ }^{3}$
}

${ }^{I}$ College of Information Science and Technology, Dalian Maritime University, Dalian, Liaoning, 116026, P.R. China;

${ }^{2}$ Dept of Information and Science, Dalian Institute of Science and Technology, Dalian, Liaoning, 116052, P.R. China;

${ }^{3}$ Software College, Dalian Jiaotong University, Dalian, Liaoning, 116045, P.R. China

\begin{abstract}
Given two points p, $\mathrm{q}$ and a sequence of $\mathrm{n}$ lines $(\mathrm{n}>1)$ in the plane, we want to find the shortest path of touring all the given lines that starts at $\mathrm{p}$ and ends at $\mathrm{q}$. In this paper, we solve the problem by reducing it to the problem of finding the shortest path that tours all the segments in a convex polygon from $p$ to $q$. We first introduce how to construct the convex polygon. Then, we propose the solution to process the intersections of two adjacent segments by crossing over two segments. Finally, based on rubber-band algorithm, a new algorithm is proposed which can find the shortest path of touring segments in a convex polygon, and the $\mathrm{O}\left(\mathrm{n}^{2}\right)$ running time can be obtained for this problem, which improves the previous $\mathrm{O}\left(\mathrm{n}^{3} \operatorname{logn}\right)$ time. Our algorithm is simple and efficient.
\end{abstract}

Keywords: Convex polygon, rubber-band algorithm, shortest path.

\section{INTRODUCTION}

Path planning is one of the central problem areas in computational geometry. The shortest touring path problem is the most classical example of path planning, for example, finding the shortest path of touring a given series of obstacles between two points in the plane [1]. In this paper, we focus on the solution to find the shortest path of touring given lines in the plane from $\mathrm{p}$ to $\mathrm{q}$. The problem is as follows.

Assume that there are two points $\mathrm{p}, \mathrm{q}$ and $\mathrm{n}>1$ line $\mathrm{l}_{\mathrm{i}}$ (for $\mathrm{i}=1,2, \ldots, \mathrm{n}$ ) in a plane $\Pi$, we want to find the shortest path that tours all the given lines $1_{i}$ from the start point $p$ to the end point q, see Fig. (1).

The problem given above is abstracted from the shortest watchman route problem, and it is also the key to solve the shortest watchman route problem $[2,3]$. The watchman route problem is derived from the well-known art gallery problem, and the goal is to find a shortest closed route in a simple polygon $\mathrm{G}$ such that one can see each point in the polygon $\mathrm{G}$ from at least one point of the route.

Hakan Jonsson has presented an algorithm for finding the shortest path that intersects $\mathrm{n}$ lines in the plane in $\mathrm{O}\left(\mathrm{n}^{5}\right)$ time [4]. No more optimal results have been reported recently. Many scholars have been studying on the touring segments problems and made some effective results. For example, for the case in which the given segments don't intersect, in 1984, D. T. Lee and F. P. Preparata proposed Funnel algorithm [5]. In 2007, Fajie Li and Reinhard Kettle proposed rubber-band algorithm $[6,7]$ (denoted by $\mathrm{R}$ algorithm) with $\mathrm{K}(\varepsilon) \cdot \mathrm{O}(\mathrm{n})$ time, where $\mathrm{K}(\varepsilon)=\left(\mathrm{L}_{0}-\mathrm{L}\right) / \varepsilon, \mathrm{L}_{0}$ is the initial

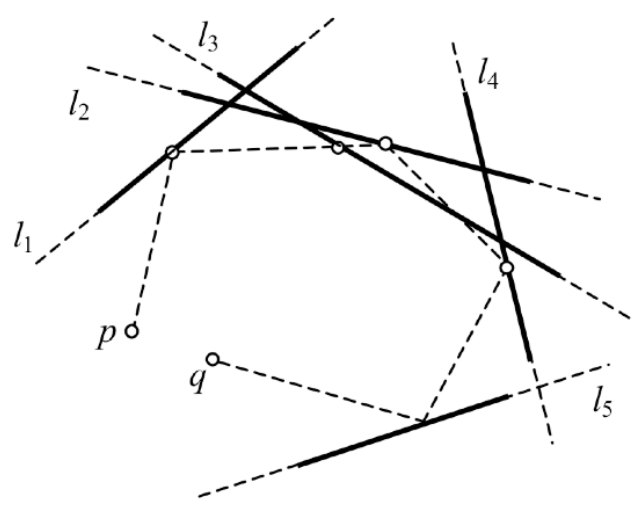

Fig. (1). The shortest path of touring 5 lines in the plane.

shortest path length of touring all segment set $\mathrm{S}, \mathrm{L}$ is the actual shortest path, and $\mathrm{n}$ is the number of segments. In 2011, R algorithm is obtained in $\mathrm{O}\left(\mathrm{n}^{2}\right)$ time by Wang and Huo by the experiment. Furthermore, they improved the algorithm and obtained the $\mathrm{O}(\mathrm{n})$ time by introducing divide and conquer into $\mathrm{R}$ algorithm [8]. For the case in which the given segments possibly intersecting, the $\mathrm{O}\left(\mathrm{n}^{3} \operatorname{logn}\right)$ time algorithm for touring a sequence of possibly intersecting line segments is known [9].

In our paper, the problem of finding the shortest path for lines can be converted to the problem of finding the shortest path for segments in a convex polygon. We first introduce how to construct the convex polygon. Then, on basis of $\mathrm{R}$ algorithm, a new algorithm is proposed which processes the degradation problem caused by the intersection by crossing over two segments when two adjacent segments intersect. Finally, we have implemented it with $\mathrm{C}++$ program and obtained the $\mathrm{O}\left(\mathrm{n}^{2}\right)$ time by experiment. The result shows that the algorithm is efficient. 


\section{THE METHOD OF CONSTRUCTING CONVEX POLYGON}

We use OPT(L) to denote the shortest path of touring given line set $\mathrm{L}$ from $\mathrm{p}$ to $\mathrm{q}$, since $\mathrm{OPT}(\mathrm{L})$ is the shortest path, the parts between consecutive points of tour $\mathrm{L}$ are line segments. Moreover, since a non-convex route in the plane is strictly longer than the boundary of its convex hull, and the convex hull of a route intersects any target line, we conclude that $\mathrm{OPT}(\mathrm{L})$ is convex [4]. It implies that OPT(L) visits each target line in one of three ways. If the angle of OPT(L) coming into a line $l_{i}$ with $l_{i}$ (incoming angle) is equal to the angle of OPT(L) going away from a line $1_{i}$ with $1_{i}$ (outgoing angle), OPT $(\mathrm{L})$ makes a perfect reflection on the line $\mathrm{l}_{\mathrm{i}}$ (see Fig. 2a). If OPT(L) passes the line $l_{i}$ twice, OPT(L) makes a crossing contact with $1_{i}$ (see Fig. 2b). The case in which OPT(L) shares a portion of lines with $l_{i}$ can be thought of a special reflection (see Fig. 2c).

Here, we respectively take the starting point $p$ and the ending point $\mathrm{q}$ as the intersection of two lines, thus the number of lines in set $\mathrm{L}$ is $\mathrm{n}+4$. We bound the region in the plane where OPT(L) visits the set $\mathrm{L}$, and consider the convex hull $\mathrm{C}$ of all crossings(see Fig. 3a). For sets $\mathrm{L}$ containing no parallel target lines, it can be shown that OPT(L) lies in C, and the point $\mathrm{p}$ and $\mathrm{q}$ lies in $\mathrm{C}$ or on the boundary of $\mathrm{C}$. However, if there are parallel lines in set L, OPT(L) may be not contained in the convex hull C (see Fig. 4). Since C is convex, each of its tangents partitions the plane into two half-planes of which the active half-plane totally contains $\mathrm{C}$. We make all tangents of $\mathrm{C}$ that are normal to target lines, denoted by the set $\mathrm{N}$, and consider the intersection $\mathrm{G}$ of the active halfplanes of all tangents in set N (see Fig. 3b), we can conclude OPT $(\mathrm{L})$ is contained in G [4]. Thus, we can reduce this problem to compute the shortest path between $p$ and $q$ which visits segments in the convex polygon G.

\section{COMPUTE THE SHORTEST PATH OF SEG- MENTS IN A CONVEX POLYGON}

For the case in which the segments in a convex polygon are disjoint, the solution has been described in detail in references [6-8]. For the case in which the segments possibly intersect, a new algorithm has been introduced as follows.

\subsection{The Method of Dealing with the Intersected Seg- ments}

When the segments intersect, suppose that the segment $\mathrm{s}_{\mathrm{i}}$ intersects with $\mathrm{s}_{\mathrm{i}+1}$ at the point $\mathrm{C}$, and $\mathrm{q}_{\mathrm{i}-1}$ is the path point on $\mathrm{s}_{\mathrm{i}-1}$ and $\mathrm{q}_{\mathrm{i}+2}$ is the path point on $\mathrm{s}_{\mathrm{i}+2}$. There are three cases between $\overline{q_{i-1} q_{i+2}}$ and the segments $\mathrm{s}_{\mathrm{i}}$ and $\mathrm{s}_{\mathrm{i}+1}$, which are as follows.

Case $1 \overline{q_{i-1} q_{i+2}}$ intersects with $\mathrm{s}_{\mathrm{i}}$ and $\mathrm{s}_{\mathrm{i}+1}$

In this case, obviously, $\overline{q_{i-1} q_{i+2}}$ is the local shortest path, denoted by $d, d=\overline{q_{i-1} q_{i+2}}$, and the order of $d$ passing through $\mathrm{s}_{\mathrm{i}}$ and $\mathrm{s}_{\mathrm{i}+1}$ is the touring order, if $d$ first passes through $\mathrm{s}_{\mathrm{i}}$, the touring order is $\mathrm{s}_{\mathrm{i}}$ and $\mathrm{s}_{\mathrm{i}+1}$, otherwise, that is $\mathrm{s}_{\mathrm{i}+1}$ and $\mathrm{s}_{\mathrm{i}}$, see Fig. (5).

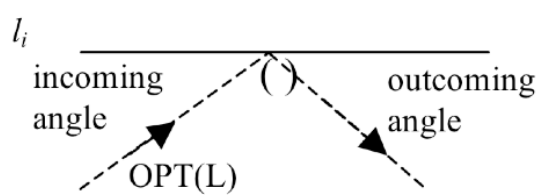

(a)

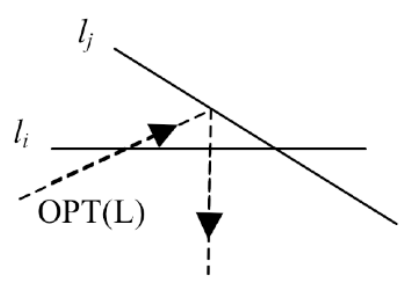

(b)

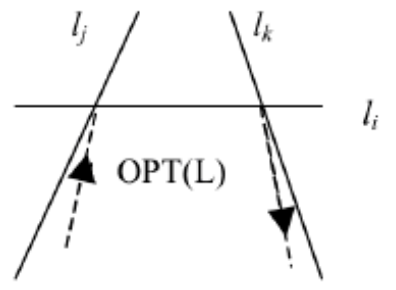

(c)

Fig. (2). Three types of contacts between OPT(L) and a line $l_{i}$.

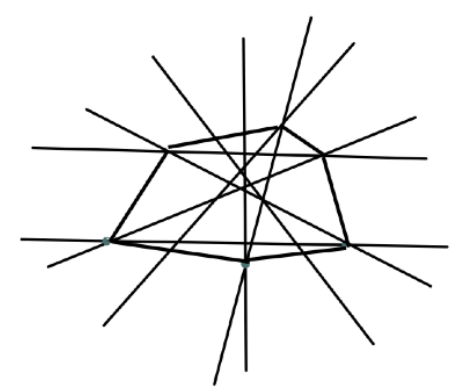

(a) The convex hull C

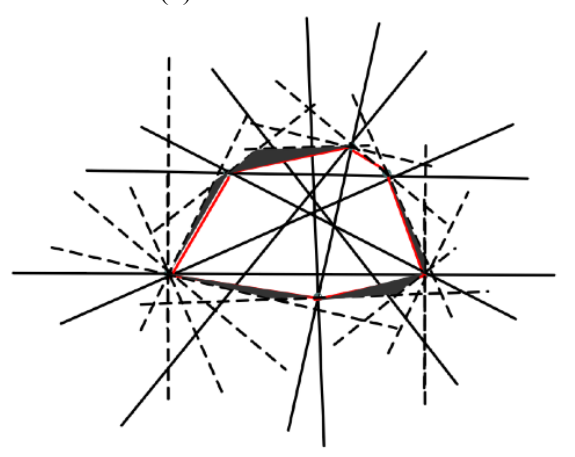

(b) The polygon $\mathrm{G}$ (shaded and containing C)

Fig. (3). Constructing convex polygon.

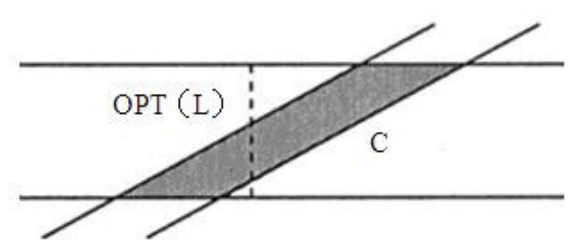

Fig. (4). OPT(L) is not contained in C. 


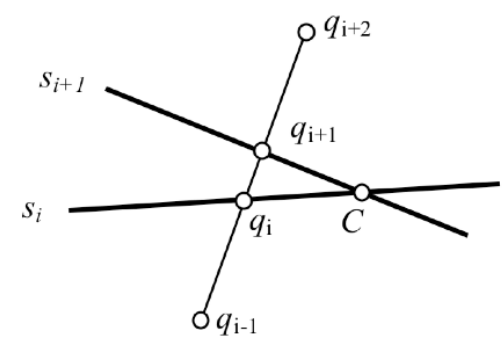

(a)

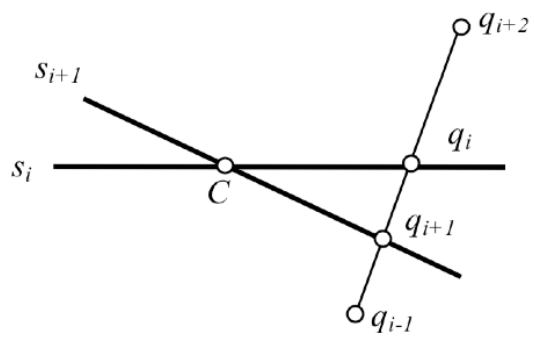

(b)

Fig. (5). $\overline{q_{i-1} q_{i+2}}$ intersects with $s_{i}$ and $s_{i+1}$.

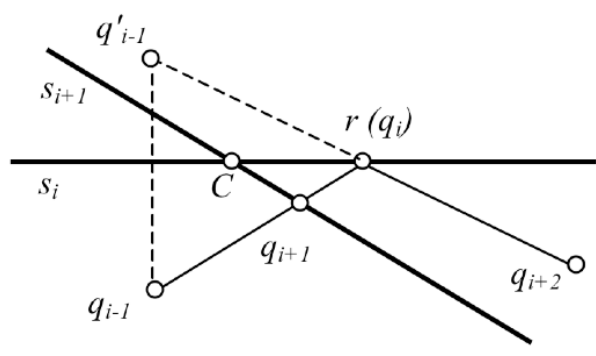

(a) Reflection point lies on $s_{i}$

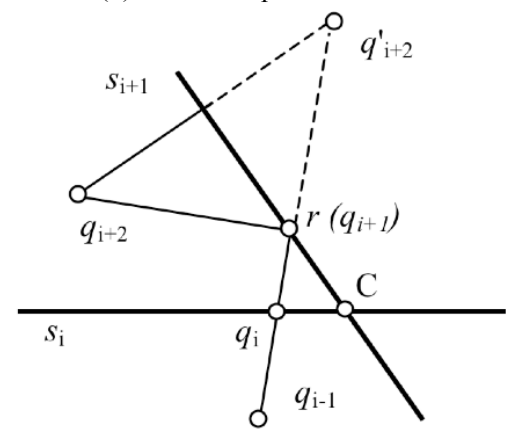

(b) Reflection point lies on $s_{i+1}$

Fig. (6). $\overline{q_{i-1} q_{i+2}}$ intersects with $s_{i}$ or $s_{i+1}$.

Case $2 \overline{q_{i-1} q_{i+2}}$ intersects with $\mathrm{s}_{\mathrm{i}}$ or $\mathrm{s}_{\mathrm{i}+1}$

In this case, to find the shortest path, computing the reflection point $r$ on the line segment $s_{i}$ or $s_{i+1}$ is needed (see Fig. 6).

The approach in the iteration process is as follows. If $q_{i-1} q_{i+2}$ only intersects with $\mathrm{s}_{\mathrm{i}}$ or $\mathrm{s}_{\mathrm{i}+1}$, the reflection point $\mathrm{r}$ lies on the segment which does not intersect with $\overline{q_{i-1} q_{i+2}}$, $d=\overline{r q_{i-1}}+\overline{r q_{i+2}}$, and the touring order is the order of $d$ passing through $\mathrm{s}_{\mathrm{i}}$ and $\mathrm{s}_{\mathrm{i}+1}$, if $d$ first passes through $\mathrm{s}_{\mathrm{i}}$, the touring order is $s_{i}$ and $s_{i+1}$, otherwise, that is $s_{i+1}$ and $s_{i}$.

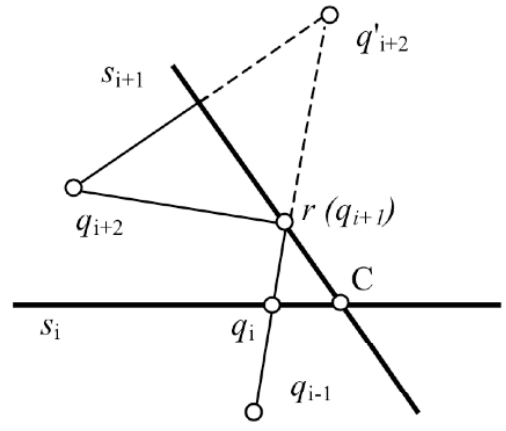

(a) Reflection point lies on $s_{i}$

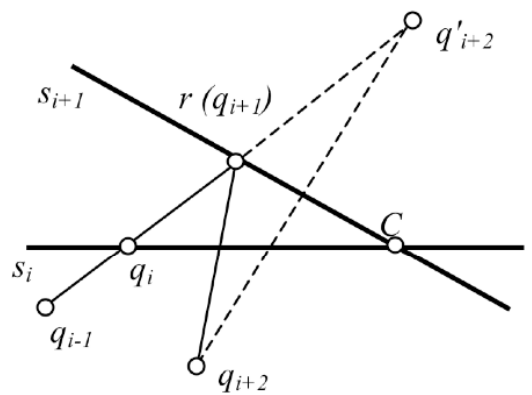

(b) Reflection point lies on $s_{i+1}$.

Fig. (7). $\overline{q_{i-1} q_{i+2}}$ doesn't intersect with $s_{i}$ and $s_{i+1}$.

Case $3 \overline{q_{i-1} q_{i+2}}$ doesn't intersect with $s_{i}$ and $s_{i+1}$

In this case, there are two cases which are shown in Figs. (7) and (8).

(1) the case of computing one reflection

The two segments can be visited by computing a reflection point (see Fig. 7). In this case, obviously, $d=\overline{r q_{i-1}}+\overline{r q_{i+2}}$, here, when $d$ passes through $\mathrm{s}_{\mathrm{i}}$ or $\mathrm{s}_{\mathrm{i}+1}$ twice, we can deal with it as above, if $d$ first passes through $s_{i}$, the touring order is $s_{i}$ and $s_{i+1}$, otherwise, that is $s_{i+1}$ and $s_{i}$.

(2) the case of computing two reflections

When the two segments can't be toured by computing a reflection point, it needs to compute two reflections. The approach is as follows. We make the reflection points, denoted by $\mathrm{q}_{\mathrm{i}-1}^{\prime}$ and $\mathrm{q}_{\mathrm{i}+2}^{\prime}$, of $\mathrm{q}_{\mathrm{i}-1}$ and $\mathrm{q}_{\mathrm{i}+2}$ with respect to $\mathrm{s}_{\mathrm{i}}$ and $\mathrm{s}_{\mathrm{i}+1}$ respectively.

In this case, there are also three situations when computing the local shortest path, see Fig. (8).

(1) if $\overline{q^{\prime}{ }_{i-1} q^{\prime}{ }_{i+2}}$ intersects with $\mathrm{s}_{\mathrm{i}}$ at the point $\mathrm{q}_{\mathrm{i}}$ and intersects with $\mathrm{s}_{\mathrm{i}+1}$ at the point $\mathrm{q}_{i+1}$, and the two path points $\mathrm{q}_{i}$ and $\mathrm{q}_{\mathrm{i}+1}$ are on the same side of $\mathrm{q}_{\mathrm{i}-1}$ and $\mathrm{q}_{\mathrm{i}+2}$, they are the two reflections and also the path points. If $\overline{q_{i-1} q_{i}}$ does not intersect with $\overline{q_{i+1} q_{i+2}}, d=\overline{q_{i-1} q_{i}}+\overline{q_{i} q_{i+1}}+\overline{q_{i+1} q_{i+2}}$ is the local shortest path, and the order of $d$ passing through $\mathrm{s}_{\mathrm{i}}$ and $\mathrm{s}_{\mathrm{i}+1}$ is the touring order (see Fig. 8a). Otherwise, we need to change the touring order of $s_{i}$ and $s_{i+1}$ in order to get the local shortest path, then $d=\overline{q_{i-1} q_{i+1}}+\overline{q_{i+1} q_{i}}+\overline{q_{i} q_{i+2}}$, see Fig. (8b). 


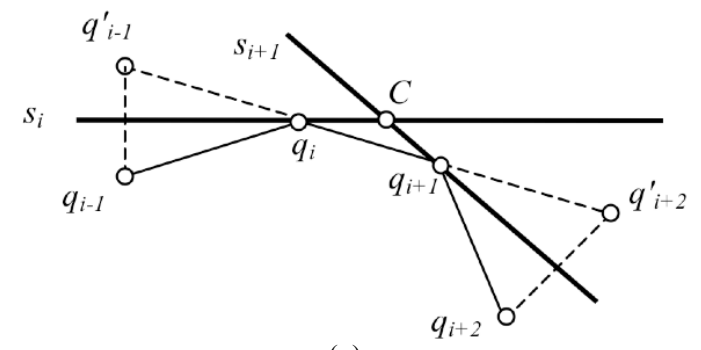

(a)

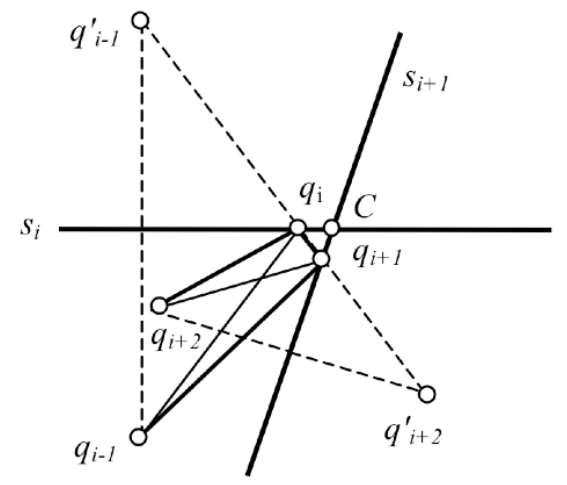

(b)

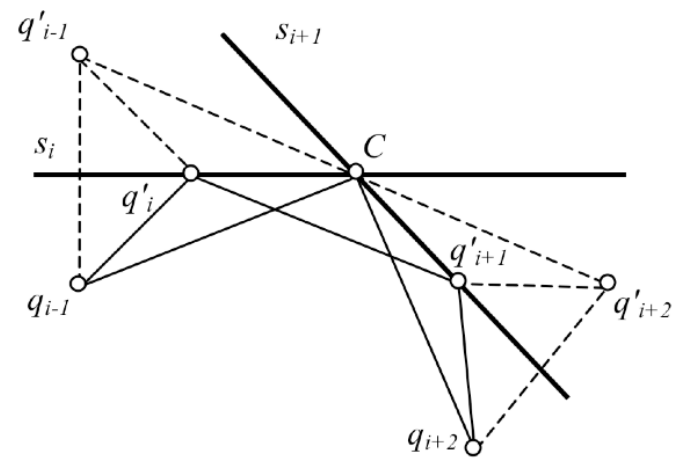

(c)

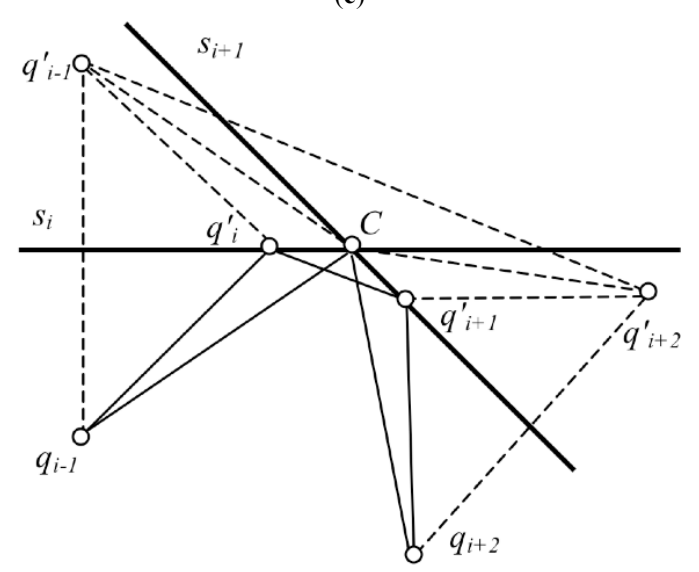

(d)

Fig. (8). $q_{i-1}$ and $q_{i+2}$ lie on the same side of $s_{i}$ and $s_{i+1}$ (two reflection points).

(2) if $\overline{q_{i-1}^{\prime} q_{i+2}^{\prime}}$ passes the point $\mathrm{C}$, the reflection points coincide with $\mathrm{C}$ and they are also the path points $\left(\mathrm{q}_{\mathrm{i}}=\mathrm{q}_{\mathrm{i}+1}=\mathrm{C}\right)$, $d=\overline{q_{i-1} C}+\overline{C q_{i+2}}$, and the order of $d$ passing through $\mathrm{s}_{\mathrm{i}}$ and $\mathrm{s}_{\mathrm{i}+1}$ is the touring order. We can make two auxiliary points $\mathrm{q}_{\mathrm{i}}^{\prime}$ and $\mathrm{q}_{\mathrm{i}+1}^{\prime}$ on $\mathrm{s}_{\mathrm{i}}$ and $\mathrm{s}_{\mathrm{i}+1}$ and prove that easily, see Fig. (8c).
(3) if $\overline{q_{i-1}^{\prime} q_{i+2}^{\prime}}$ doesn't pass the point $\mathrm{C}$ and $\overline{q_{i-1}^{\prime} q_{i+2}^{\prime}}$ is on the different side of $\overline{q_{i-1} C}$ and $\overline{C q_{i+2}}, d=\overline{q_{i-1} C}+\overline{C q_{i+2}}$, the path points $\mathrm{q}_{\mathrm{i}}$ and $\mathrm{q}_{\mathrm{i}+1}$ also coincide with $\mathrm{C}$, and the order of $d$ passing through $\mathrm{s}_{\mathrm{i}}$ and $\mathrm{s}_{\mathrm{i}+1}$ is the touring order, see Fig. 8d). It can be proved as follows. In Fig. (8d), we select a point $\mathrm{q}_{\mathrm{i}}^{\prime}$ on segment $\mathrm{s}_{\mathrm{i}}$ and a point $\mathrm{q}_{\mathrm{i}+1}^{\prime}$ on $\mathrm{s}_{\mathrm{i}+1}$, since $\overline{q_{i-1}^{\prime} q_{i}^{\prime}}+\overline{q_{i}^{\prime} q_{i+1}^{\prime}}+\overline{q_{i+1}^{\prime} q_{i+2}^{\prime}}=\overline{q_{i-1} q_{i}^{\prime}}+\overline{q_{i}^{\prime} q_{i+1}^{\prime}}+$ $\overline{q_{i+1}^{\prime} q_{i+2}}, \quad \overline{C q_{i-1}^{\prime}}+\overline{C q_{i+2}^{\prime}}=\overline{C q_{i-1}}+\overline{C q_{i+2}}$, obviously, $\overline{q^{\prime}{ }_{i+2} q_{i+1}^{\prime}}+\overline{q_{i+1}^{\prime} q_{i}^{\prime}}+\overline{q_{i}^{\prime} q_{i-1}^{\prime}} \quad>\overline{C q_{i+2}^{\prime}}+\overline{C q_{i-1}^{\prime}}$, then $\overline{q_{i+2} q_{i+1}^{\prime}}+\overline{q_{i+1}^{\prime} q_{i}^{\prime}}+\overline{q_{i}^{\prime} q_{i-1}}>\quad \overline{C q_{i+2}}+\overline{C q_{i-1}}, \quad d=$ $\overline{C q_{i+2}}+\overline{C q_{i-1}}$ follows.

\subsection{Algorithm}

In this paper, on basis of $\mathrm{R}$ algorithm, we apply a new algorithm to compute the shortest touring path, with crossing over two segments to process the intersection of them when these two segments intersect. The algorithm is as follows. Assume that $s_{i}$ interests with $s_{i+1}$, we can compute the path point $\mathrm{q}_{\mathrm{i}}$ on $\mathrm{s}_{\mathrm{i}}$ and the path point $\mathrm{q}_{\mathrm{i}+1}$ on $\mathrm{s}_{\mathrm{i}+1}$ according to the position relation between points and segments descried above. Next, we judge the position relationship of $s_{i+1}$ and $\mathrm{s}_{\mathrm{i}+2}$, if $s_{i+1} \cap s_{i+2}=\varnothing$, we compute the path point $\mathrm{q}_{\mathrm{i}+2}$ on $\mathrm{s}_{\mathrm{i}+2}$ and $\mathrm{q}_{\mathrm{i}+3}$ on $\mathrm{s}_{\mathrm{i}+3}$. Otherwise, we compute the path point $\mathrm{q}_{\mathrm{i}+1}$ on $\mathrm{s}_{\mathrm{i}+1}$ and $\mathrm{q}_{\mathrm{i}+2}$ on $\mathrm{s}_{\mathrm{i}+2}$.

The algorithm is composed of four functions which are Main function, UpdatePoint function, SkipOne function and SkipTwo function. Main function calculates the shortest path by calculating all the iteration process. UpdatePoint function calculates the shortest path in every iteration. SkipOne function calculates the shortest path with crossing over one segment. SkipTwo function calculates the shortest path with crossing over two segments. SkipTwo function is the key to the algorithm. In reference [8], Main function, UpdatePoint function, SkipOne function have been described in detail. Here, we mainly give the SkipTwo function, it is described below.

SkipTwo $\left(q_{i-1}, s_{i}, s_{i+1}, q_{i+2}\right)$ function.

In this function, $s_{i}$ and $s_{i+1}$ denote segments, $q_{i-1}$ and $q_{i+2}$ denote path points calculated on segments $s_{i-1}$ and $s_{i+2}$ respectively. The function is used to calculate the local optimal path of touring $s_{i}$ and $s_{i+1}$ from $q_{i-1}$ to $q_{i+2}$, and the path points $\mathrm{q}_{\mathrm{i}}$ and $\mathrm{q}_{\mathrm{i}+1}$ can be obtained.

Denote by $r=\overline{q_{i-1} q_{i+2}}$, L the path calculated by SkipOne function, and $s_{i}$ intersected with $s_{i+1}$ at q.

Case $1 q_{i-1} q_{i+2}$ intersects with $\mathrm{s}_{\mathrm{i}}$ and $\mathrm{s}_{\mathrm{i}+1}$

If $\left(r \cap s_{i} \neq \varnothing \& \& \quad r \cap s_{i+1} \neq \varnothing\right)$, we consider that $s_{i}$ intersects with $r$ at $c_{1}$ and $s_{i+1}$ intersects with $r$ at $c_{2}$ respectively, let $q_{i}=c_{1}$ and $q_{i+1}=c_{2}$, and if $\left(\left|\overline{q_{i-1} r_{2}}\right|<\left|\overline{q_{i-1} r_{1}}\right|\right)$, call change $\left(s_{i}, s_{i+1}\right)$ and call change $\left(q_{i}, q_{i+1}\right)$.

Case $2 \overline{q_{i-1} q_{i+2}}$ intersects with $s_{i}$ or $s_{i+1}$ 


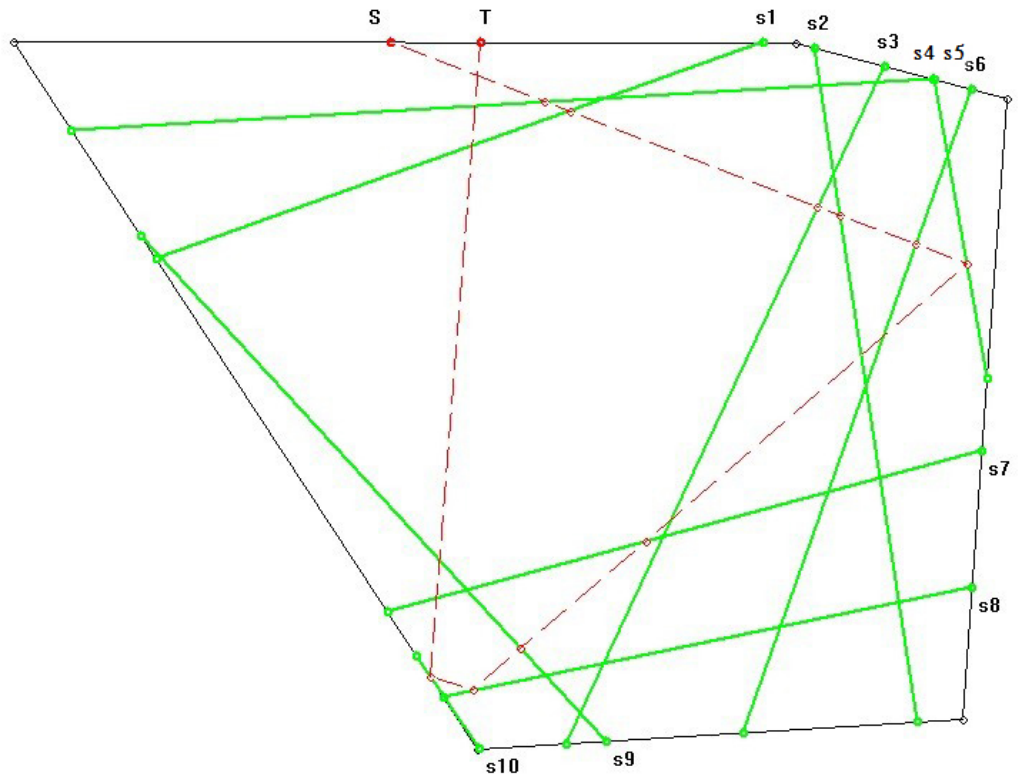

Fig. (9). The running result of 10 segments in a convex polygon.

if $\left(r \cap s_{i} \neq \varnothing \| r \cap S_{i+1} \neq \varnothing\right)$

if $\left(r \cap s_{i} \neq \varnothing\right)$, call SkipOne $\left(q_{i-1}, s_{i+1}, q_{i+2}\right)$, and we consider that $s_{i}$ intersects with $L$ at $c_{1}$ and $s_{i+1}$ intersects with $L$ at $c_{2}$ respectively.

Let $q_{i}=c_{1}, q_{i+1}=c_{2}$ and $r \operatorname{Tmp}=\overline{c_{2} q_{i+2}}$,

if $\left(r \operatorname{Tmp} \cap s_{i} \neq \varnothing\right)$, call chang $\left(s_{i}, s_{i+1}\right)$,

and call change $\left(q_{i}, q_{i+1}\right)$.

else call SkipOne $\left(q_{i-1}, s_{i}, q_{i+2}\right)$,we consider that $s_{i}$ intersects with $L$ at $c_{1}$ and $s_{i+1}$ intersects with $L$ at $c_{2}$ respectively.

Let $q_{i}=c_{1}, q_{i+1}=c_{2}$ and $r \operatorname{Tmp}=\overline{c_{1} q_{i-1}}$.

if $\left(r \operatorname{Tmp} \cap s_{i+1} \neq \varnothing\right)$ call change $\left(s_{i}, s_{i+1}\right)$, and call change $\left(q_{i}, q_{i+1}\right)$.

Case $3 \overline{q_{i-1} q_{i+2}}$ doesn't intersect with $s_{i}$ and $s_{i+1}$

Call SkipOne $\left(q_{i-1}, s_{i}, q_{i+2}\right)$. Denote by $L$ intersecting with $s_{i}$ at $c_{1}$ and $r \operatorname{Tmp}=\overline{c_{1} q_{i-1}}$.

if ( $\left.r \operatorname{Tmp} \cap s_{i+1} \neq \varnothing\right)$, and the intersection is $c_{2}$, then let $q_{i}=c_{1}, q_{i+1}=c_{2}$, call change $\left(s_{i}, s_{i+1}\right)$ and call change $\left(q_{i}, q_{i+1}\right)$.

else call SkipOne $\left(q_{i-1}, s_{i+1}, q_{i+2}\right)$. Denote by $L$ intersecting with $s_{i+1}$ at $c_{2}$, and $r \operatorname{Tmp}=\overline{r_{2} q_{i+2}}$.

if $\left(r \operatorname{Tmp} \cap s_{i} \neq \varnothing\right)$, and $r \operatorname{Tmp}$ intersects with $s_{i}$ at $c_{1}$, else we compute the reflection points, denoted by $q_{i-1}^{\prime}$ and $q_{i+2}^{\prime}$, of $q_{i-1}$ and $q_{i+2}$ with respect to $s_{i}$ and $s_{i+1}$ respectively, let $r \operatorname{Tmp}=\overline{q_{i-1}^{\prime} q_{i+2}^{\prime}}$, and denote $s_{i}$ intersecting with $r \operatorname{Tmp}$ at $r_{1}$ and $s_{i+1}$ intersecting with $r$ Tmp at $r_{2}$.

if ( $q$ and $q_{i-1}$ lie on the same side of rTmp), let $q_{i+1}=q_{i}=q$.

else let $q_{i}=c_{1}$ and $q_{i+1}=c_{2}$.

if $\left(\overline{r_{1} q_{i-1}} \cap \overline{q_{i+1} q_{i+2}} \neq \varnothing\right)$, call change $\left(s_{i}, s_{i+1}\right)$ and call change $\left(q_{i}, q_{i+1}\right)$.

\section{THE ANALYSIS OF RUNNING RESULT}

In this paper, we have implemented the algorithm with $\mathrm{C}++$ program. Here, we give an example of touring $10 \mathrm{seg}-$ ments in a convex polygon. In Fig. (9), the solid lines are the segments which the lines intersect with the convex polygon, and the intersections are the endpoints of segments. The path shown in dotted lines is the shortest path of touring the 10 lines from $\mathrm{S}$ to $\mathrm{T}$.

We can see that the order of segments is from $s_{1}$ to $s_{10}$, while the output order is different from that of input. Moreover, the three cases of path points and segments described above have been contained. $q_{1}, s_{3}, s_{2}, q_{6}$ is the case 1 , $q_{2}, s_{6}, s_{5}, q_{7}$ is the case 2 , and $q_{7}, s_{8}, s_{10}, T$ is the case 3 .

The running time of the algorithm is complex and difficult to analyze, so in this paper, we adopt the method of analysis by running time counting to analyze the algorithm's time complexity. We have tested the algorithm applying a large of segment sets (for example 500), and the segments number is 1000 in every segment set. Based on the results, we have got the fitting curve equation of the algorithm, that is $y=1 E-05 x^{2}-0.011 x+3.810$, see Fig. (10). The results show that the algorithm is in $\mathrm{O}\left(\mathrm{n}^{2}\right)$ time, and it is superior to 


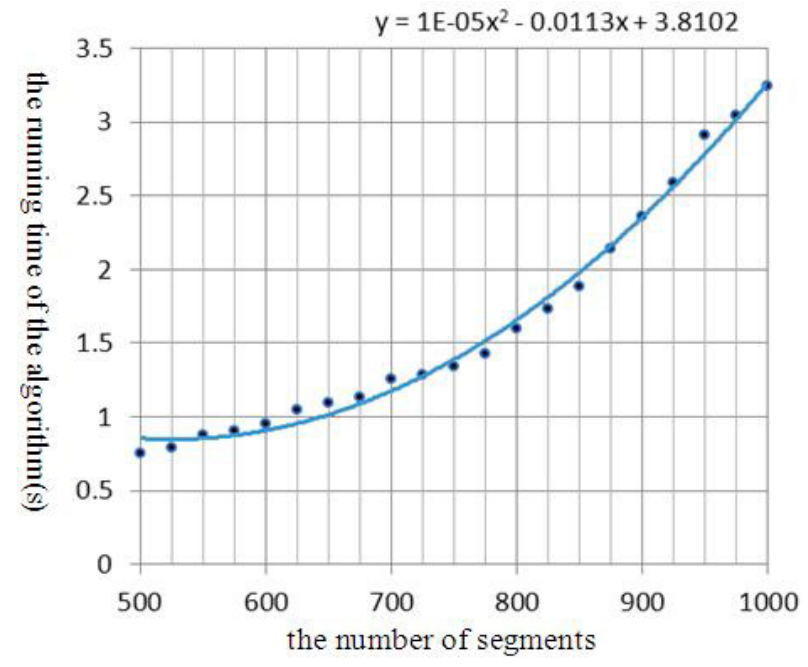

Fig. (10). The fitting curve equation of the algorithm.

the $\mathrm{O}\left(\mathrm{n}^{3} \operatorname{logn}\right)$ time of reference [9]. It can be seen that our algorithm is efficient.

\section{CONCLUSION}

In this paper, we convert the problem of computing the shortest touring path for lines to the problem of computing the shortest touring path for segments in a convex polygon, and we present an algorithm for computing the shortest touring path of segments in a convex polygon, especially, we have implemented it. The result shows that our algorithm is efficient.

This research has made preliminary results. If the segments haven't been given the order, it has been proved to be a NP hard problem, if we study the shortest path of touring rays, which is open and lies in between the problems concerning lines and segments. Those problems need to be further studied.

\section{CONFLICT OF INTEREST}

The authors confirm that this article content has no conflict of interest.

\section{ACKNOWLEDGEMENTS}

This work is supported by the National Natural Science Foundation of China (No. 61173034, 61272171), the General Project of Liaoning Province Education Department (No. L2014183) and the General Project of Dalian Institute of Science and Technology (No. KYZ1416).

\section{REFERENCES}

[1] M. de Berg, O. Cheong, M. van Kreveld, and M. Overmars, Computational Geometry: Algoriths and Applications, ( ${ }^{\text {rd }}$ ed.), Springer-Verlag Publishers: Berlin, 2009, pp. 10-11.

[2] X. Tan, "Fast computation of shortest watchman routes in simple polygons", Information Processing Letters, vol. 77, no. 1, pp. 27 33, 2001.

[3] A. Dumitrescu, J. S. B. Mitchell, and P.. Żyliński, "Watchman route for line and segments", Algorithm Theory - SWAT 2012 Lecture Notes in Computer Science, vol. 7357, pp. 36-47, 2012.

[4] H. Jonsson, "The traveling salesman problem for lines in the plane", Information Processing Letters, vol. 82, pp. 137-142, 2002.

[5] D. T. Lee, and F. P. Preparata, "Euclidean shortest paths in the presence of rectilinear barriers", Networks, vol. 14, no. 3, pp. 393410, 1984.

[6] F. Li, and R. Klette, "Exact and approximate algorithms for the calculation of shortest paths", IMA Journal of Management Mathematics, vol. 17, no. 1, pp. 134-138, 2006.

[7] L. Fa-jie, and K. Reinhard, "Shortest path algorithms for sequences of polygons", CAAI Transactions on Intelligent Systems, vol. 3, no 1, pp. 23-30, 2008.

[8] L. Wang, L. Huo, and D. He, "An improved algorithm for euclidean shortest paths of visiting line segments in the plane", Journal of Convergence Information Technology, vol. 6, no. 6, pp. 119-125, 2011.

[9] M. Dror, A. Efrat, A. Lubiw and J.S.B. Mitchell, "Touring a sequence of polygons", In: Proceedings $35^{\text {th }}$ Annual ACM Symposium Theory Computation, California, USA, 2003, pp. 473-482.

\begin{tabular}{lcc}
\hline Received: September 16, 2014 & Revised: December 23, 2014 & Accepted: December 31, 2014 \\
(C) Wang et al.; Licensee Bentham Open. & &
\end{tabular}

This is an open access article licensed under the terms of the Creative Commons Attribution Non-Commercial License (http://creativecommons.org/licenses/by-nc/3.0/) which permits unrestricted, non-commercial use, distribution and reproduction in any medium, provided the work is properly cited. 